\title{
Complexity in the Management of Brainstem Gliomas: Our Experience
}

\author{
Murugaiyan Nagarajan, Athisayaraj Patrick Joshua, Ramesh Banu \\ Department of Radiation Oncology, Valavadi Narayanasamy Cancer Center, G. Kuppuswamy Naidu Memorial Hospital, Coimbatore, \\ Tamil Nadu, India.
}

\section{Corresponding Author: \\ Dr. Murugaiyan Nagarajan \\ Email:mnr81@yahoo.com}

This is an Open Access article distributed under the terms of the Creative Commons Attribution License (creativecommons.org/ licenses/by/3.0).

Received Accepted

Published

March 11, 2019

October 9, 2019

November 5, 2019

\begin{abstract}
Background: Brainstem gliomas (BSGs) constitutes 10\% of pediatric and $2 \%$ of adult brain tumors. Overall management of BSGs is complex due to lack of biopsy, location of the tumor making it inoperable and dosimetric constraints in RT delivery. We did a retrospective analysis on clinical outcomes of BSGs in our institute. Case Series: Seven cases of BSGs (5 adults and 2 pediatric) were included. Most common presenting symptom was weakness of limbs. Magnetic Resonance Imaging was the sole imaging modality. Biopsy was feasible in two patients. Median RT dose was 50 Gy. Two patients received concurrent temozolamide and one patient received adjuvant temozolamide. Median overall survival was 7.9 months. In our cohort adults had better survival than children. Conclusion: BSGs are distinct group of brain tumors. In most of the cases biopsy is not feasible and MRI is the current standard diagnostic imaging modality. Radiotherapy is the standard treatment options for BSGs.
\end{abstract}

Keywords: Brainstem, Biopsy, Glioma, Magnetic Resonance Imaging, Pons.

\section{Introduction}

Brainstem glioma (BSG) constitutes $10 \%$ of brain tumors in pediatric population [1]. It is rare in adults constituting $<2 \%$ of gliomas [2,3]. Many retrospective series suggested better survival in adults than in children. The most possible reason for this difference is due the histological nature of the tumor. Majority of the adult BSGs are low grade when compared to that of pediatric BSGs [4].

Role of surgery in the treatment of BSGs is limited, radiotherapy (RT) is an essential treatment modality in BSGs. Management of BSGs is complex due to many factors. In most of the cases biopsy is not possible. Surgery is not feasible due to its eloquent location. Further in view of dosimetric constraints RT dose escalation is not feasible in many cases. In view of complexity in the management of BSGs, we did this study in order to understand the clinical outcomes in BSGs following RT in our institution.

\section{Case Series}

We reviewed the records of patients with BSGs from the year 2013-2018 who were treated in our radiotherapy department. Patients who had the tumor epicenter, defined as the centre of tumor bulk in the brainstem were included in our study. Their complete medical records and treatment data were obtained and analyzed. Overall survival is calculated from the date of diagnosis to date of last follow up and the status of the patient during last follow up was noted.

We identified seven patients with BSGs treated in our institute. Four patients (57\%) were males and three patients $(43 \%)$ were females. Two patients belonged to pediatric age group. Median age was 40 years (Range: 7-72 years). Most common presentation was weakness of limbs $(42.8 \%)$ followed by visual disturbances (28.5\%). $71.4 \%$ of the patients had short duration of symptoms (less than three months) whereas 
$28.6 \%$ had symptom duration for more than three months. Longest duration of symptom was 12 months. MRI was the imaging modality for all the patients. Two patients had stereotactic biopsy whereas five patients were treated without biopsy. All the patients received RT as definitive treatment. Two patients received concurrent temozolamide along with RT and one patient received adjuvant temozolamide for 6 months. Median overall survival was 7.9 months. During follow up, six patients expired following RT and one patient is still alive without any evidence of disease after 32 months follow up.

\section{Discussion}

Brain stem gliomas are heterogenous tumors with variable biology and outcomes. In our case cohort, we treated five adult and two pediatric BSGs. Similarly in a study conducted by Yoshida et al. [5], 14 pediatric and 16 adult BSGs were treated. Fisher et al. [6] in their study found that pons $(59 \%)$ was the most common site of BSGs followed by midbrain (14\%) as seen in our study. Also it was found that patients with tumor epicenter at midbrain had better prognosis than at pons. But in our study there were no significant difference in terms of survival.

It has been found that patients who had short interval of symptoms ( $<3$ months) had poor outcomes [7]. In contrast, in our study there was no such association. Further $71.4 \%$ of the patients had short interval of symptoms ( $<3$ months) and 28.6\% of the patients had duration of symptom $>3$ months. MRI was the sole imaging modality for diagnosis in our case cohort. $70 \%$ of the patients showed T2 hyper-intense lesions. Most of the lesions showed no contrast enhancement. $57 \%$ of the lesions showed iso or hypo-intense in T1 sequence. This finding is in concordant with literature $[4,8,9]$. Role of MRI is important to determine the nature of the lesion. Yin and Zhang in their study compared the radiological and histopathological correlation of BSGs. In their study, the accuracy of diagnosing

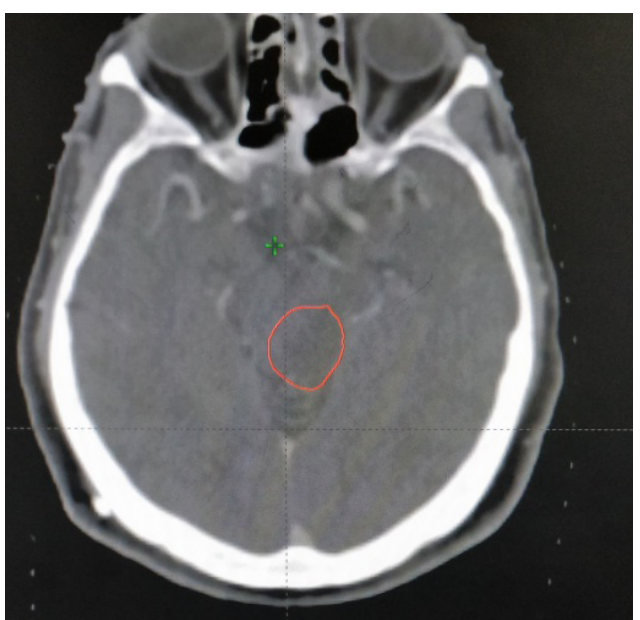

Fig.1: Computed Tomography axial section showing tumor in the midbrain before radiotherapy.

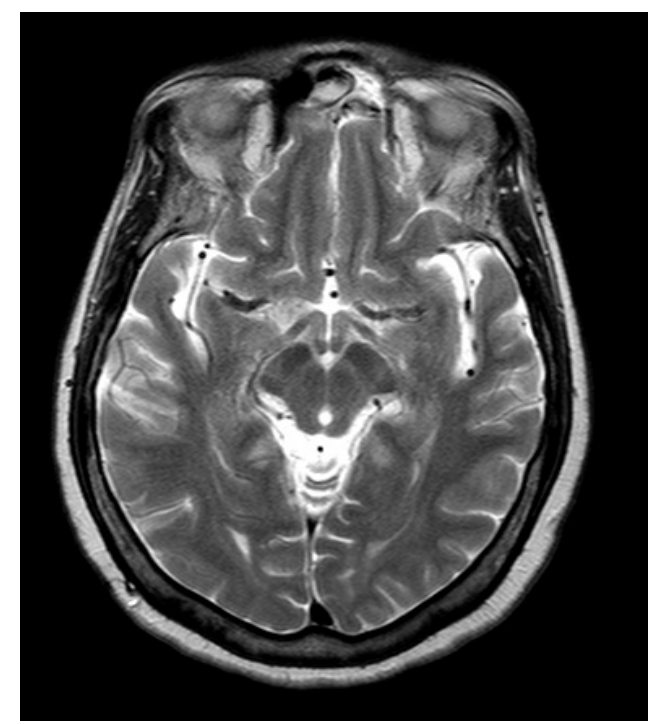

Fig.2: T2 weighted axial section Magnetic Resonance Imaging showing no tumor six months after radiotherapy.

BSGs based on MRI was $95.3 \%$. Out of 150 patients diagnosed to have BSGs only 7 patients had non BSG tumors. MRI alone has higher predictive value in diagnosing BSGs [10].

Benefit of biopsy should be weighed against the morbidity associated with it. Though there are several studies that have shown that stereotactic biopsy is feasible with less morbidity and mortality [11], the role of biopsy in BSGs is not clearly defined. Hence role of pre-treatment biopsy is still 
controversial. In our study, only two (29\%) patients had biopsy of the lesion. Biopsy was deferred in other cases as it was not feasible. $72 \%$ of the patients were treated with RT without biopsy. Yoshida et al. [6] in their study found that biopsy was feasible in $7 \%$ of pediatric patients and $38 \%$ of the adults which is similar to our study. Similarly $63 \%$ of the patients were treated with RT without biopsy which is consistent with our findings. Molecular analysis of gliomas have therapeutic implications. But in BSGs it is still under evaluation. Karremann et al. [12] found that mutation in H3K27M in children is associated with worse prognosis. In our present study, molecular analysis was not done for the patients who underwent biopsy. Unless the clinical picture is atypical, surgery is not indicated in diffuse pontine pediatric glioma [13]. In view of morbidity of surgery, it is contraindicated in diffuse intrinsic pontine gliomas. However in adult BSGs surgery can be performed in tectal or exophytic medullary gliomas. Grade III and IV tumors had significant tumor progression and worse survival despite adequate surgery and subtotal resection offered no benefit in survival [14].

RT is the essential therapeutic modality for treatment of BSGs. Median dose of RT was 50 Gy in our study. Brainstem is an eloquent area. Hence dose escalation is often not feasible. Though previous studies have found no severe brainstem toxicity in BSGs, these studies are limited by short median survival. Hence long term side effects of higher dose of RT in BSGs are not reported in the literature. Many RT schedules were evaluated in the past varing from hyper-fractionated to hypo-fractionated RT [15-17]. In our case series, a 9 year old child with diffuse intrinsic pontine glioma was treated with RT. At presentation he had right hemiplegia and bulbar palsy with ECOG performance status 4 . He received RT to brain $30 \mathrm{~Gy} / 15 \#$ followed by $19.8 \mathrm{~Gy} / 11$ \# boost to the tumor. Post RT he had significant improvement in power on right side and his performance status improved to 2. Though RT can cause significant tumor shrinkage, it is not curative in most of the cases.
Table 1: Profile of patients.

\begin{tabular}{|l|l|}
\hline Characteristics & Number (Percentage) \\
\hline Median Age (Range) & 40 years (7-72) years \\
\hline Sex & $4(57 \%)$ \\
Male & $3(43 \%)$ \\
\hline Semale & $1(15 \%)$ \\
Site of tumor & $6(85 \%)$ \\
Midbrain & - \\
Mens & \\
\hline Symptoms & $3(43 \%)$ \\
Paresis & $2(29 \%)$ \\
Visual disturbance & $1(14 \%)$ \\
Giddiness & $1(14 \%)$ \\
Headache & \\
\hline Duration of symptoms & $5(71 \%)$ \\
Less than 3 months & $2(29 \%)$ \\
More than 3 months & \\
\hline Treatment & $6(86 \%)$ \\
Radiotherapy alone & - \\
Chemotherapy alone & $1(14 \%)$ \\
Radiotherapy+ & \\
Chemotherapy & 7.9 months \\
\hline Median overall survival & \\
\hline
\end{tabular}

Combination of chemotherapeutic agents with RT had been found to be beneficial in the treatment of gliomas. However evidence for this approach is not available in adult BSGs. In children, previous retrospective studies found no significant survival advantage with addition of temozolamide $[18,19]$. In our study cohort, two adult patients received concurrent temozolamide out of which one patient received adjuvant temozolamide for six cycles. Median overall survival (OS) in our study cohort was 7.6 months (Range: 2 months-32.7 months). This result is in concordant with previous studies [5,17].

\section{Conclusion}

Management of BSGs is complex due to lack of biopsy, lack of feasibility for surgery and dose escalation impossibility. Radiotherapy is the 
standard treatment of BSGs. Adult BSGs have slightly improved outcomes than pediatric BSGs. MRI is the imaging modality for diagnosis in the absence of biopsy. Newer molecular approaches are required to improve survival in future.

Contributors: MN: concept and manuscript review. PJ: literature search, data acquisition and manuscript preparation; RB: definition of intellectual contents, design and manuscript editing. MN will act as guarantor. All authors approved the final version of this manuscript.

Funding: None; Competing interests: None stated.

\section{References}

1. Ostrom QT, Gittleman H, Liao P, Rouse C, Chen Y, Dowling $\mathrm{J}$, et al. CBTRUS statistical report: primary brain and central nervous system tumors diagnosed in the United States in 2007-2011. Neuro-oncology. 2014;16(suppl_4):1-63.

2. White HH. Brain stem tumors occurring in adults. Neurology. 1963;13(4):292-300.

3. Hargrave D, Bartels U, Bouffet E. Diffuse brainstem glioma in children: critical review of clinical trials. The Lancet Oncology. 2006;7(3):241-248.

4. Laigle-Donadey F, Doz F, Delattre JY. Brainstem gliomas in children and adults. Current Opinion in Oncology. 2008;20(6):662-667.

5. Yoshida K, Sulaiman NS, Miyawaki D, Ejima Y, Nishimura $\mathrm{H}$, Ishihara $\mathrm{T}$, et al. Radiotherapy for brainstem gliomas in children and adults: A single institution experience and literature review. Asia Pacific Journal of Clinical Oncology. 2017;13(2):e153-160.

6. Fisher PG, Breiter SN, Carson BS, Wharam MD, Williams JA, Weingart JD. A clinicopathologic reappraisal of brain stem tumor classification: Identification of pilocytic astrocytoma and fibrillary astrocytoma as distinct entities. Cancer. 2000;89(7):1569-1576.

7. Lachi PK, Irrakula M, Ahmed SF, Joseph D, Pamidighantam S, Naidu KV. Clinical profile and outcomes in brainstem glioma: an institutional experience. Asian Journal of Neurosurgery. 2015;10(4):298.

8. Donaldson SS, Laningham F, Fisher PG. Advances toward an understanding of brainstem gliomas. Journal of Clinical Oncology. 2006;24(8):1266-1272.

9. Jallo GI, Biser-Rohrbaugh A, Freed D. Brainstem gliomas. Child's Nervous System. 2004;20(3):143-153.

10. Yin L, Zhang L. Correlation between MRI findings and histological diagnosis of brainstem glioma. Canadian
Journal of Neurological Sciences. 2013;40(3):348-354.

11. Manoj N, Arivazhagan A, Bhat DI, Arvinda HR, Mahadevan A, Santosh V. Stereotactic biopsy of brainstem lesions: techniques, efficacy, safety, and disease variation between adults and children: a single institutional series and review. Journal of Neurosciences in Rural Practice. 2014;5(1):32.

12. Karremann M, Gielen GH, Hoffmann M, Wiese M, Colditz N, Warmuth-Metz M, et al. Diffuse highgrade gliomas with $\mathrm{H} 3 \mathrm{~K} 27 \mathrm{M}$ mutations carry a dismal prognosis independent of tumor location. Neurooncology. 2017;20(1):123-131.

13. Albright AL, Packer RJ, Zimmerman R, Rorke LB, Boyett J, Hammond GD. Magnetic resonance scans should replace biopsies for the diagnosis of diffuse brain stem gliomas: a report from the Children's Cancer Group. Neurosurgery. 1993;33(6):1026-1030.

14. Majchrzak K, Bobek-Billewicz B, Hebda A, Majchrzak H, Ładziński P, Krawczyk L. Surgical treatment and prognosis of adult patients with brainstem gliomas. Neurologia I Neurochirurgia Polska. 2018;52(5):623633.

15. Freeman CR, Krischer J, Sanford RA, Burger PC, Cohen M, Norris D. Hyperfractionated radiotherapy in brain stem tumors: results of a Pediatric Oncology Group study. International Journal of Radiation Oncology Biology Physics. 1988;15(2):311-318.

16. Packer RJ, Boyett JM, Zimmerman RA, Albright AL, Kaplan AM, Rorke LB, et al. Outcome of children with brain stem gliomas after treatment with $7800 \mathrm{cGy}$ of hyperfractionated radiotherapy. A children's cancer group phase 1/11 trial. Cancer. 1994;74(6):1827-1834.

17. Janssens GO, Jansen MH, Lauwers SJ, Nowak PJ, Oldenburger FR, Bouffet E, et al. Hypofractionation vs conventional radiation therapy for newly diagnosed diffuse intrinsic pontine glioma: a matched-cohort analysis. International Journal of Radiation Oncology Biology Physics. 2013;85(2):315-320.

18. Cohen KJ, Heideman RL, Zhou T, Holmes EJ, Lavey RS, Bouffet E. Temozolomide in the treatment of children with newly diagnosed diffuse intrinsic pontine gliomas: a report from the Children's Oncology Group. Neuro-oncology. 2011;13(4):410-416.

19. Chassot A, Canale S, Varlet P, Puget S, Roujeau T, Negretti L. Radiotherapy with concurrent and adjuvant temozolomide in children with newly diagnosed diffuse intrinsic pontine glioma. Journal of Neuro-oncology. 2012;106(2):399-407. 